\title{
Proportion of medication error reporting and associated factors among nurses: a cross sectional study
}

\author{
Abebaw Jember ${ }^{1 *}$, Mignote Hailu', Anteneh Messele ${ }^{2}$, Tesfaye Demeke ${ }^{3}$ and Mohammed Hassen ${ }^{1}$
}

\begin{abstract}
Background: A medication error (ME) is any preventable event that may cause or lead to inappropriate medication use or patient harm. Voluntary reporting has a principal role in appreciating the extent and impact of medication errors. Thus, exploration of the proportion of medication error reporting and associated factors among nurses is important to inform service providers and program implementers so as to improve the quality of the healthcare services.

Methods: Institution based quantitative cross-sectional study was conducted among 397 nurses from March 6 to May 10, 2015. Stratified sampling followed by simple random sampling technique was used to select the study participants. The data were collected using structured self-administered questionnaire which was adopted from studies conducted in Australia and Jordan. A pilot study was carried out to validate the questionnaire before data collection for this study. Bivariate and multivariate logistic regression models were fitted to identify factors associated with the proportion of medication error reporting among nurses. An adjusted odds ratio with 95\% confidence interval was computed to determine the level of significance.
\end{abstract}

Result: The proportion of medication error reporting among nurses was found to be $57.4 \%$. Regression analysis showed that sex, marital status, having made a medication error and medication error experience were significantly associated with medication error reporting.

Conclusion: The proportion of medication error reporting among nurses in this study was found to be higher than other studies.

Keywords: Medication error, Medication error reporting, Federal Level Governmental Hospital, Nurse

\section{Background}

Medication therapy is the most common intervention prescribed for healthcare consumers [1] and safe medication administration represents one of the routine, highly complex and essential nursing care responsibilities [2]. Medication administration is amongst the potentially hazardous nursing tasks in hospitals because of liability to errors [3]. The reporting of error incidents and specific phases of healthcare delivery such as the safe use of medications can improve the safety of patients [4].

A single medication error (ME) may prolong hospital stay or even end up in death. This affects the quality and

\footnotetext{
* Correspondence: abebaw5360@gmail.com

'Department of Medical Nursing, School of Nursing, College of Medicine and Health Sciences, University of Gondar, Gondar, Ethiopia

Full list of author information is available at the end of the article
}

continuity of the healthcare services [5] by affecting patient safety which is an important indicator of healthcare quality and encompasses various nursing care procedures. Executing a ME has been seen to be psychologically devastating to the nurse and harmful to the patient [6].

Globally, MEs present a substantial contribution to ill health, and even death and are listed as one of the five medical error categories classified by the American Institute of Medicine [7]. Out of estimated patients' deaths of 6000 to 20,000 each year from medical errors in Taiwan, $10 \%$ of medical lawsuits were because of MEs where the majority of the errors were grossly underreported [8]. A prospective cross-sectional study conducted in an intensive care unit of a specialized hospital in Ethiopia showed a ME of $51.8 \%$ [9]. 
Nevertheless how huge or insignificant the incidence, it is difficult to have a general concept of MEs in less developed and developing countries due to inefficient documentation and error-reporting systems and insufficient research in the area [7]. Analysis and appreciation of the root causes of MEs allows developing complex medication error prevention mechanisms [10] which improve patient safety.

\section{Literature review}

A medication error is any preventable event that may cause or lead to inappropriate medication use or patient harm [11]. Medication error is a global issue where $5 \%$ of the MEs are deadly and almost $50 \%$ are preventable [12]. Medication error reporting is one of the major issues in today's health care and prevention is linked to accurate reporting of errors [7]. Voluntary reporting is indispensable to appreciate the extent and impact of MEs [13]. Nurses' interception of $86 \%$ of the MEs was presented in a descriptive crosssectional study conducted in one large medical center hospital in southern Taiwan with sample size of 597 nurses using self-administered questionnaires and the significance of error reporting was given a weight as intercepting [8]. Moreover, consideration of nurses' perceived barriers to medication error reporting (MER) is a crucial step to strengthen medication safety [8] and it was shown in a study that more than $90 \%$ of healthcare consumers believe that errors should be reported [3].

\section{Incidence and prevalence of medication errors}

Medication errors which are made during prescription, dispensing and administration [14] are common and preventable causes of patient harm [15]. Precise figure of the incidence and prevalence of MEs is difficult to obtain because the rate varies from study to study [7]. Studies showed a range of rate of serious patient injuries due to medication errors as 1 to $2 \%$ [16], 9 to 13\% [2], 29\% [17], and as high of 51.8\% [9], and estimated $30.5 \%$ deaths per year in a survey in the United States of America (USA) were attributable to MEs [15]. A study conducted in Southern Iran with the purpose of determining the frequency of MEs in an emergency department of a teaching hospital revealed that $96.5 \%$ of patients had experienced at least one medication error, making the rate of errors 3.5 per patient [18]. A descriptive survey of 300 nurses working in hospitals affiliated to Iran University of Medical Sciences using stratified multistage sampling disclosed a mean of 19.5 medication errors that the nurses acknowledged within 3-months period however the mean of error reporting was only 1.3 of error cases $[19,20]$.

\section{Factors related to medication error reporting}

A focus group study on barriers to MER in Canada identified barriers as an individual, organizational and cultural [21]. According to a descriptive cross-sectional study with sample size of 799 nurses conducted in Jordan, the proportion of medication error reporting was relatively high among female nurses than male nurses [7]. Twenty-six percent of nurses in a study conducted in Israel indicated that all MEs in their wards were reported and $46 \%$ of the nurses showed self-reporting of MEs. The nurses emphasized on a personal barrier to non-reporting such as ME experiences and error reporting experiences [22].

A perception that incidence reports do not result in significant changes or benefits and errors that did not result in harm were among the factors that affected the attitude of nurses to report medication errors [23, 24]. Perceived barriers which affect attitude of nurses to report medication errors were fear of adverse consequences from reporting and being subjected to disciplinary actions, fear of being blamed, fear of reaction from the nurse manager, from peers and fear of loss of jobs $[6-8,19,25]$. Other barriers for not reporting MEs include nurses not being aware that an error had occurred, process of reporting (detailed paperwork, time constraints, not understanding incident reporting process), forgetting to make a report when the ward is busy, lack of time for reporting and lack of awareness of the importance of reporting $[19,23,26]$.

Two-thirds $(66.7 \%)$ of nurses involved in a study conducted in two state hospitals in Turkey who stated that they involved in medication errors in the preceding 6 months had not reported the errors. The reported reasons (social factors) for not reporting MEs included fear of consequences, fear of a culture of blame and the need to cover up for the colleague involved [27].

Modifiable barriers to MER for nurses reported in different studies as organizational factors were revealed as lack of feedback to the reporter, lack of a readily available MER system, lack of information on how to report a ME, no positive feedback for giving medication correctly, too much emphasis on ME as a quality indicator of nursing care and motivational factors (such as no encouragement by management, fear of loss of professional registration), lack of organizational leadership and support $[3,8,19,25,28]$. Similarly taking medical responsibility and fear of distrust from patients were barriers of medication error reporting [29].

Encouraging administrative attitudes and responses to MER were appreciated in a study to enhance nurses' voluntary reporting [30]. It is indicated in the literature that strategies should be implemented to establish reporting mechanisms to reduce medication errors at national as well as international levels [31]. Establishing structured protocols on drug administration and adopting a non- 
punitive approach to reporting medication errors were shown to decrease medication errors and improve patient safety [32]. Proportion of medication error reporting by nurses might be affected by multiple factors such as socio-demographic, social, attitude of nurses, and organizational factors [7, 8, 12, 19] (Fig. 1 indicates the factors involved in medication error reporting).

Health care systems in most developing countries suffer from serious deficiencies in quality, equity, efficiency and financing [33]. The quality of care is evaluated in the light of the provider's technical standards and clients' expectations. Medication administration is among the routine and highly complex nursing care activities which plays a great role in patient care and outcome. Medication safety and medication errors are important concerns for healthcare consumers, health-care professionals, researchers and policy makers worldwide.

Nurses are more prone to making medication errors because of the increasing demands and pressures placed on them. Critical incidents must be detected and reported and turned into positive situations, from which lessons are learned and used to design better patient care practices and systems. So far nurses are at the front line of defense to intercept and report medication errors, yet the errors are severely under-detected and underreported in practice.

Medication errors affect the quality of health care delivery. Improving patient safety and learning from errors relies on voluntary error reporting which gives the complete picture of medication errors. Thus, exploration of the proportion of nurses reporting medication errors and associated factors is important to inform service providers, program implementers and policy makers to improve the quality of the healthcare service.

\section{Aim}

The aim of this study was to assess the proportion of medication error reporting and to explore the relationships among the barriers; socio-demographic factors, organizational factors, social factors, and attitude of nurses.

Incident reporting represents more appropriate information about incidents and can detect preventable events if it is supported within the clinical settings. However, many of the incidents are not reported or are simply not recognized and yet it has been shown in the literature that nurses predominantly report incidents compared to other healthcare professionals [34]. Conversely, studies have demonstrated under-reporting of MEs among nurses [35].

\section{Methods}

An institution based quantitative cross-sectional study was conducted at three Federal Ministry of Health level governmental hospitals located in the nation's capital Addis Ababa from March 6 to May 10, 2015. There are four hospitals under the FMoH; Alert, St. Peter, St. Paul and Amanuel. Amanuel Mental Specialized Hospital is the only referral mental hospital in the country which delivers counseling and treatment for patients. St. Paul's Millennium Medical College Hospital delivers medical services for an annual average of 200,000 patients. St. Peter's TB Specialized Hospital has been giving services related to tuberculosis treatment. Alert Hospital was excluded because of protocol issues.

\section{Sample size determination}

A single population proportion formula was used to calculate the sample size:

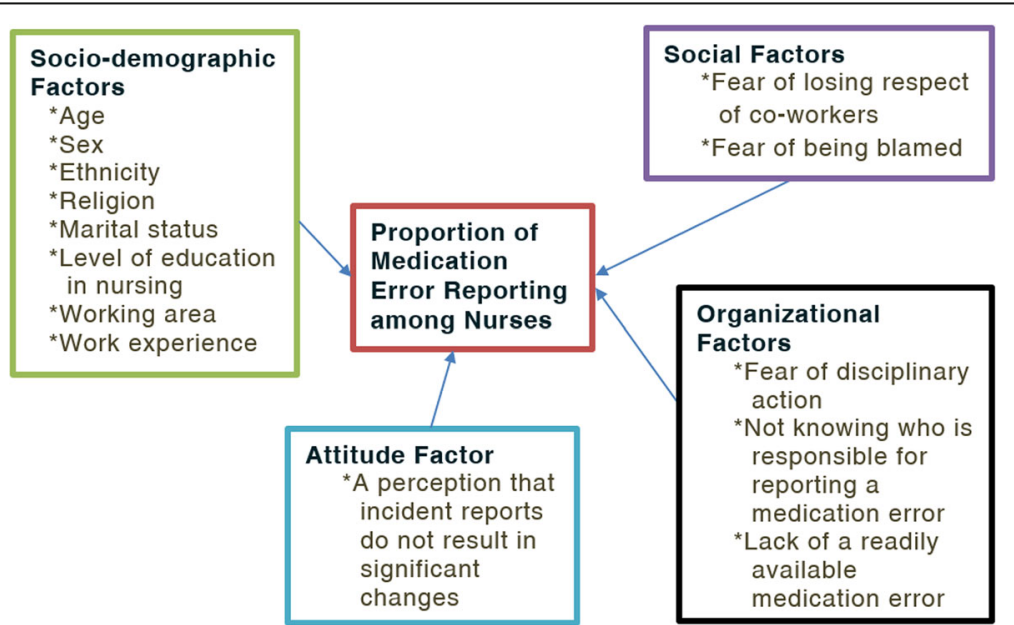

Fig. 1 Conceptual framework of the factors related to the proportion of medication error reporting. [Source: Prepared by the investigator after thorough searching of literature] 


$$
n=\frac{Z^{2} \alpha / 2^{p(1-p)}}{d^{2}}
$$

Where: $\mathrm{n}=$ minimum sample size required for the study.

$\mathrm{z}=$ score for $95 \%$ confidence interval $(\mathrm{z}=1.96)$.

$\mathrm{p}=$ prevalence of medication error reporting $(50 \%)$.

$\mathrm{d}=$ tolerable error $(\mathrm{d}=5 \%)$.

Since the proportion of medication error reporting in Ethiopia is unknown, $50 \%$ prevalence of medication error reporting was taken:

$$
\begin{aligned}
& n_{o}=\frac{(1.96)^{2} 0.5(1-0.5)}{0.05^{2}} \\
& n_{o}=\frac{0.9604}{0.0025}=384.16 \approx 385
\end{aligned}
$$

For possible non-response, the sample size was increased by $10 \%$, thus comprising 38.416 respondents the final sample size became 423 .

\section{Sampling procedure}

Stratified sampling technique was used to allocate the sample proportionally to each $\mathrm{FMoH}$ level governmental hospital and then the study participants who had work experience of six months and above were selected from the list of all nurses using simple random sampling technique; a lottery method (Fig. 2).

\section{Data collection tool and procedure}

Data were collected by three nurses who were diploma holders using structured self-administered Englishversion questionnaire. The data collection tool was adopted from two studies conducted in Australia by Evans [3] and in Jordan by Mrayyan et al. [7] with the authors' permissions and face and content validity were established by two expert nurse advisors. The tool was composed of five parts. The first part contained sociodemographic characteristics of nurses. The second part of the questionnaire was related to error incidence. The third part related to medication error reporting was used to estimate the proportion of medication error reporting. The fourth and fifth parts were used to collect data regarding the attitudes of nurses on medication error reporting and perceived organizational culture and reality of dealing with errors respectively (Additional file 1).

\section{Data quality control}

Data quality was assured by conducting a pretest among $22(5 \%)$ nurses at Zewditu hospital and appropriate modifications were made after analyzing the pre-test result before the actual data collection. The questionnaire

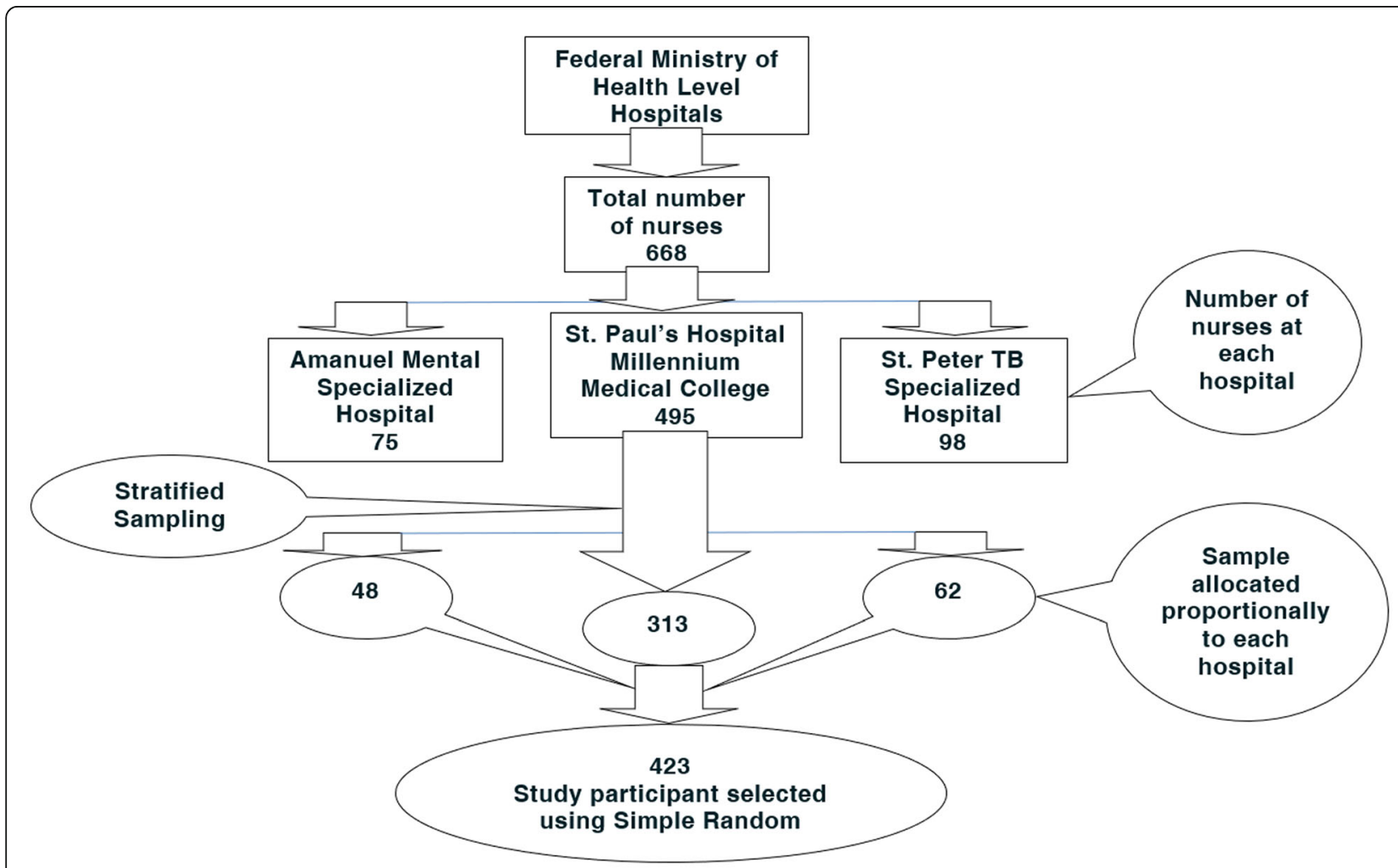

Fig. 2 Schematic presentation of the sampling procedure to select the study participants 
was coded before data collection and cross-checked for consistency and completeness every day.

\section{Data management and analysis}

The returned questionnaires were checked for completeness, cleaned and the data were entered into EPI Info-7 and then exported to SPSS (Statistical Package for the Social Sciences) version 20.0 for analysis. Frequencies and cross tabulations were used to summarize descriptive statistics and tables were used for data presentation. Binary logistic regression was used to identify factors associated with medication error reporting and then the variables were checked for significant association using $p$-value, odds ratio and 95\% confidence interval.

\section{Result}

Socio-demographic characteristics

Out of 423 proposed study participants, 403 participated in the study giving a response rate of $95.27 \%$. Six $(1.41 \%)$ of the returned questionnaires were found to be incomplete and excluded and the rest 20 (4.72\%) of nurses chose not to participate in the study. 397 (93.85\%) of study participants' responses were analyzed. The majority (53.7\%) of respondents were male. The mean $(+$ standard deviation) age of the respondents was $28.37(+5.55)$ years. 307 (77.3\%) respondents were Orthodox Christian followed by Protestant 48 (12.1\%), 208 (52.4\%) of the respondents were Amhara in their ethnic background and 291 (73.3\%) of the participants were single. On the other hand, 154 $(38.8 \%)$ of the respondents worked in internal medicine wards and level of education of 237 (59.7\%) of the participants was Bachelor of Science in Nursing. Moreover, 182 $(45.8 \%)$ of the respondents served $1-3$ years in the nursing profession (Table 1).

\section{Proportion of medication error reporting}

The proportion of medication error reporting among nurses in this study was found to be $57.4 \%(n=288)$. Out of the total participants $(n=397)$, relatively high (74.5\%) proportion of medication error reporting was found among female nurses as compared to male nurses (42.7\%). A high proportion (70.8\%) of medication error reporting was disclosed by married participants than participants who are single (52.6\%). On the other hand, 277 (69.8\%) of the participants perceived that medication errors should be reported as they occur. Moreover, (70.7\%) of the nurses who did not make errors themselves reported medication errors.

\section{Factors associated with medication error reporting}

In bivariate logistic regression analysis sex, marital status, having made a medication error, medication error experience, and working area were associated with medication error reporting. However, in multivariate analysis
Table 1 Demographic characteristics of nurses at federal level teaching hospitals, Addis Ababa $2015(n=397)$

\begin{tabular}{|c|c|}
\hline Variables & $\mathrm{n}(\%)$ \\
\hline \multicolumn{2}{|l|}{ Sex } \\
\hline Male & $213(53.7)$ \\
\hline Female & $184(46.3)$ \\
\hline \multicolumn{2}{|l|}{ Age } \\
\hline $19-29$ & 312 (78.6) \\
\hline $30-39$ & $52(13.1)$ \\
\hline $40-49$ & $21(5.3)$ \\
\hline $50-59$ & $12(3.1)$ \\
\hline \multicolumn{2}{|l|}{ Ethnicity } \\
\hline Amhara & $208(52.4)$ \\
\hline Oromia & $93(23.4)$ \\
\hline SNNPR & $60(15.1)$ \\
\hline Tigray & $20(5.0)$ \\
\hline Others $^{a}$ & $16(4.0)$ \\
\hline \multicolumn{2}{|l|}{ Religion } \\
\hline Orthodox & $307(77.3)$ \\
\hline Protestant & $48(12.1)$ \\
\hline Muslim & $36(9.1)$ \\
\hline Catholic & $6(1.5)$ \\
\hline \multicolumn{2}{|l|}{ Marital Status } \\
\hline Single $e^{b}$ & $291(73.3)$ \\
\hline Married & $106(26.7)$ \\
\hline \multicolumn{2}{|c|}{ Level of education in nursing } \\
\hline $\mathrm{BSC}$ & $237(59.7)$ \\
\hline Diploma & $160(40.3)$ \\
\hline \multicolumn{2}{|l|}{ Working area } \\
\hline Internal medicine ward & $154(38.8)$ \\
\hline Surgical ward & $73(18.4)$ \\
\hline Emergency room & $63(15.9)$ \\
\hline Psychiatry & $47(11.8)$ \\
\hline Intensive care unit & $31(7.8)$ \\
\hline Pediatric ward & $29(7.3)$ \\
\hline \multicolumn{2}{|c|}{ Service year in the nursing profession } \\
\hline$<1$ & 54 (13.6) \\
\hline $1-3$ & $182(45.8)$ \\
\hline $4-5$ & 67 (16.9) \\
\hline $6-10$ & 62 (15.6) \\
\hline$>10$ & $32(8.1)$ \\
\hline
\end{tabular}

athers: Benishangul-Gumuz, Harari, Gambella

${ }^{\mathrm{b}}$ Single includes divorced and widowed

sex, marital status, having made a medication error and medication error experience were associated with medication error reporting (Table 2). 
Table 2 Bivariate and multivariate logistic regression analysis of factors associated with proportion of medication error reporting among nurses at selected Federal Ministry of Health level hospitals, Addis Ababa $2015(n=397)$

\begin{tabular}{|c|c|c|c|c|}
\hline \multirow[t]{2}{*}{ Variables } & \multicolumn{2}{|c|}{ Medication error reporting practice } & \multicolumn{2}{|l|}{ Odds Ratio (95\% Cl) } \\
\hline & Yes & No & Crude & Adjusted \\
\hline \multicolumn{5}{|l|}{ Sex } \\
\hline Male & 91 & 122 & 1.00 & 1.00 \\
\hline Female & 137 & 47 & $0.256(0.167-0.393)$ & $0.273(0.165-0.450)^{*}$ \\
\hline \multicolumn{5}{|c|}{ Marital status } \\
\hline Single & 153 & 138 & 1.00 & 1.00 \\
\hline Married & 75 & 31 & $0.458(0.284-0.739)$ & $0.454(0.251-0.821)^{*}$ \\
\hline \multicolumn{5}{|c|}{ Medication error experience } \\
\hline Yes & 94 & 104 & 1.00 & 1.00 \\
\hline No & 134 & 65 & $0.438(0.292-0.659)$ & $0.445(0.274-0.722)^{*}$ \\
\hline \multicolumn{5}{|c|}{ I made medication errors } \\
\hline Yes & 170 & 145 & 1.00 & 1.00 \\
\hline No & 58 & 24 & $0.485(0.287-0.820)$ & $0.426(0.230-0.789)^{*}$ \\
\hline \multicolumn{5}{|c|}{ Should errors be reported } \\
\hline Yes & 132 & 145 & 1.00 & 1.00 \\
\hline No & 96 & 24 & $0.228(0.137-0.377)$ & $0.151(0.082-0.277)^{*}$ \\
\hline \multicolumn{5}{|c|}{ Error reporting leads to beneficial and constructive activity } \\
\hline Yes & 197 & 159 & 1.00 & 1.00 \\
\hline No & 31 & 10 & $0.400(0.190-0.840)$ & $0.881(0.334-2.322)$ \\
\hline \multicolumn{5}{|c|}{ Working area: Intensive care unit } \\
\hline Yes & 39 & 24 & 1.00 & 1.00 \\
\hline No & 8 & 23 & $4.672(1.804-12.101)$ & $4.471(0.502-13.307)$ \\
\hline \multicolumn{5}{|c|}{ Religion: Protestant } \\
\hline Yes & 165 & 142 & 1.00 & 1.00 \\
\hline No & 34 & 14 & $0.478(0.247-0.927)$ & $0.672(0.296-1.525)$ \\
\hline
\end{tabular}

"Statistically significant at $P<0.05$

The proportion of medication error reporting was high among female nurses as compared to male nurses. Female nurses were $72.7 \%$ times more likely to report medication errors than male nurses $(\mathrm{AOR}=0.273$; 95\% $\mathrm{CI}=0.165-0.450)$. Similarly, marital status was an important predictor of medication error reporting. Nurses who are married were $54.6 \%$ times less likely to report medication errors as compared to those who are single $(\mathrm{AOR}=0.454 ; 95 \% \mathrm{CI}=0.251-0.821)$.

Having a medication error experience was found to be another important determinant of medication error reporting. Nurses who had no medication error experience were $55.5 \%$ times more likely to report medication errors than those who had medication error experiences $(\mathrm{AOR}=0.445 ; 95 \% \mathrm{CI}=0.274-0.722)$. The odds of medication error reporting among nurses who have not made errors themselves was $57.4 \%$ times higher as compared to those nurses who had previously made medication errors $(\mathrm{AOR}=0.426 ; 95 \% \mathrm{CI}=0.230-0.789)$.

\section{Discussion}

The proportion of medication error reporting among nurses was found to be $57.4 \%$. This finding is slightly higher as compared to studies in Jordan 42.1\% [7], Australia 41.9\% [3] and California, USA 28.9\% [6]. The possible differences could be related to the differences in organizational medication error reporting systems and differences in the time frame that the studies are bounded.

In this study medication error experience of nurses was significantly associated with medication error reporting. Nurses who had previous experiences of medication errors and their reporting processes that errors made by others were $55.5 \%$ less likely to report error incidents than those who did not have error experiences and those nurses who had previously made medication errors were $57.4 \%$ less likely to report error incidents than those who did not make medication errors. This result is consistent with the result from Israel [22] and South Korea [26] that ME and having made 
errors hindered nurses from reporting MEs. However, this result was inconsistent with other results from Taiwan [8], Southern Taiwan [29] and Saudi Arabia [25]. The difference may be related to the severity of medication errors since minor errors are less likely to be reported, even though it was difficult to determine the effect of medication errors experienced regarding severity.

Most of the participants (69.8\%) perceived that errors should be reported as they occur for the safety of patients and this was consistent with the study from the United Kingdom [23] however lower than the study from Taiwan $(87.7 \%)$ [8]. The possible difference may be due to the lack of a readily available practice system of medication error reporting.

In this study, the proportion of female nurses who reported medication errors was higher than the male nurses and was statistically significant. The result was similar with that of the study from Jordan [7]. In contrast to other studies, marital status in this study was statistically significant with error reporting $(\mathrm{AOR}=$ $0.454 ; 95 \% \mathrm{CI}=0.251-0.821)$ showing that nurses who are married were $54.6 \%$ less likely to report medication errors. This might be related to barriers like fear of disciplinary actions since it was put in second place as perceived barrier to reporting by nurses though not statistically significant.

\section{Conclusion}

The proportion of medication error reporting among nurses in this study was found to be high. Medication error experience, having made a medication error, sex of participants and marital status were significantly associated.

\section{Limitation}

Causes of medication errors were not studied in this research. Knowing the causes of medication errors gives a complete picture of medication errors. Therefore, it would be better if the study was triangulated. Response bias and recall bias were not reduced.

\section{Recommendation \\ To Federal Ministry of Health}

It is recommended that the Federal Ministry of Health identify and address gaps in medication error identification and reporting regarding the establishment of the reporting system to improve patient safety.

\section{To the respected hospitals}

The respective hospitals efforts will be significant in identifying and addressing gaps in medication error reporting among nurses and creating a conducive environment for the establishment and maintenance of efficient reporting system. A condition in which reported errors are changed into opportunities to learn from them.

\section{To the respected nurses}

Nurses' contribution to the documentation of errors and reporting is an important input for the smooth provision of quality care and improved patient outcome.

\section{To researchers}

Further investigation, qualitative in nature, should be made in order to have a complete picture of medication errors in Ethiopia.

\section{Additional file}

Additional file 1: Questionnaire Medication Error Reporting. Questionnaire. Questionnaire prepared to collect data for the assessment of the intent of nurses to report medication errors and associated factors at federal level governmental teaching hospitals in Addis Ababa, Ethiopia 2015. (DOCX 26 kb)

\section{Abbreviations}

FMoH: Federal Ministry of Health; ME: Medication error; MER: Medication error reporting; $\mathrm{MoH}^{\prime}$ : Ministry of Health; USA: United States of America

\section{Acknowledgements}

We would like to express our deepest and heartfelt gratitude to the University of Gondar for funding the research work. Our gratitude further goes to the respective hospitals, Amanuel Mental Specialized Hospital, St. Paul's Hospital Millennium Medical College, and St. Peter's TB Specialized Hospital, and the nurses who chose to participate in this study.

\section{Funding}

This work was supported by the University of Gondar by funding the expenses of the research work.

Availability of data and materials

The datasets generated and analyzed during this study are available from the corresponding author on reasonable request.

\section{Authors' contributions}

AJF: Theorized the research problem, designed the study, conducted field work, analyzed the data and revised the manuscript. MHG: Involved in the supervision of the field work, revision of the research design, data analysis and revision of the manuscript for publication. AMB: Involved in the supervision of the field work, revision of the research design, data analysis and revision of the manuscript for publication. TDA: Involved in the supervision of the field work, revision of the research design, data analysis and revision of the manuscript for publication. MHS: Contributed in the revision of the research design, statistical analysis, and preparation of the manuscript. All authors read and approved the final manuscript.

\section{Ethics approval and consent to participate}

Ethical clearance and approval was obtained from Ethical Review Committee of School of Nursing, College of Medicine and Health Sciences, University of Gondar and permission was obtained from the Ethical Review Committees of each respective hospital.

Consent form was put as a first page of each questionnaire, and included the name of the researcher, the purpose of the study, and a number of ethically based instructions. Participants were assured that their involvement in the study was after having been informed about the study without undue influence and could withdraw from the study at any time without the need to give reason. The privacy of the participants was maintained while they fill the questionnaire and confidentiality of the participants was maintained by keeping anonymity and keeping the data only accessible by the investigator. Although there may not be immediate and direct benefits for the participants, nurses were informed of the benefits from the nursing knowledge gained through the process. The participants were informed that there were no financial benefits for participating in the research, no potential harms that impact on employment, or social status, the utilization of the gathered data to be used only for the intended research, 
and the publication of the results of the study in a reputable journal with no identifiable information that links to the participants.

\section{Consent for publication}

Not applicable.

\section{Competing interests}

The authors declare that they have no competing interests.

\section{Publisher's Note}

Springer Nature remains neutral with regard to jurisdictional claims in published maps and institutional affiliations.

\section{Author details}

${ }^{1}$ Department of Medical Nursing, School of Nursing, College of Medicine and Health Sciences, University of Gondar, Gondar, Ethiopia. ${ }^{2}$ Unit of Community Health Nursing, School of Nursing, College of Medicine and Health Sciences, University of Gondar, Gondar, Ethiopia. ${ }^{3}$ Department of Pediatric and Child Health Nursing, School of Nursing, College of Medicine and Health Sciences, University of Gondar, Gondar, Ethiopia.

Received: 23 November 2016 Accepted: 6 March 2018

Published online: 12 March 2018

\section{References}

1. Wakefield DS, Wakefield BJ, Uden-Holman T, Borders T, Blegen M, Vaughn T. Understanding why medication administration errors may not be reported. Am J Med Qual. 1999;14(2):81-8.

2. Anselmi ML, Peduzzi $M$, dos Santos CB. Errors in the administration of intravenous medication in Brazilian hospitals. J Clin Nurs. 2007;16(10): 1839-47.

3. Evans SM. Attitudes and barriers to incident reporting: a collaborative hospital study. Qual Saf Health Care. 2006;15(1):39-43.

4. Gaal S, Verstappen W, Wensing M. Patient safety in primary care: a survey of general practitioners in the Netherlands. BMC Health Serv Res. 2010;10(1):21.

5. Zeleke A, Chanie T, Woldie M. Medication prescribing errors and associated factors at the pediatric wards of Dessie referral hospital, Northeast Ethiopia. Int Arch Med. 2014;7(1):18

6. Ulanimo VM, O'Leary-Kelley C, Connolly PM. Nurses' perceptions of causes of medication errors and barriers to reporting. J Nurs Care Qual. 2007;22(1):28-33.

7. Mrayyan MT, Shishani K, Al-Faouri I. Rate, causes and reporting of medication errors in Jordan: nurses? Perspectives. J Nurs Manag. 2007;15(6):659-70.

8. Chiang $\mathrm{H}-\mathrm{Y}$, Pepper GA. Barriers to nurses' reporting of medication administration errors in Taiwan. J Nurs Scholarsh. 2006:38(4):392-9.

9. Agalu A, Ayele Y, Bedada W, Woldie M. Medication administration errors in an intensive care unit in Ethiopia. Int Arch Med. 2012:5(1):15.

10. Montesi $\mathrm{G}$, Lechi A. Prevention of medication errors: detection and audit. $\mathrm{Br}$ J Clin Pharmacol. 2009:67(6):651-5.

11. Banning M. Medication errors: professional issues and concerns. Nurs Older People. 2006;18(3):27-32.

12. Nuckols TK, Bell DS, Liu H, Paddock SM, Hilborne LH. Rates and types of events reported to established incident reporting systems in two US hospitals. Qual Saf Health Care. 2007;16(3):164-8.

13. Halbesleben JRB, Wakefield BJ, Wakefield DS, Cooper LB. Nurse burnout and patient safety outcomes nurse safety perception versus reporting behavior. West J Nurs Res. 2008;30(5):560-77.

14. Keers RN, Williams SD, Cooke J, Ashcroft DM. Prevalence and nature of medication administration errors in health care settings: a systematic review of direct observational evidence. Ann Pharmacother. 2013:47(2):237-56.

15. Haw C, Stubbs J, Dickens GL. Barriers to the reporting of medication administration errors and near misses: an interview study of nurses at a psychiatric hospital. J Psychiatr Ment Health Nurs. 2014;21(9):797-805.

16. Nebeker JR, Barach P, Samore MH. Clarifying adverse drug events: a Clinician's guide to terminology, documentation, and reporting. Ann Intern Med. 2004; 140(10):795-801.

17. Donchin Y, Gopher D, Olin M, Badihi Y, Biesky M, Sprung CL, et al. A look into the nature and causes of human errors in the intensive care unit. Qual Saf Health Care. 2003;12(2):143-7.

18. Vazin A, Zamani Z, Hatam N. Frequency of medication errors in an emergency department of a large teaching hospital in southern Iran. Drug Healthc Patient Saf. 2014;6:179-84.
19. Joolaee S, Hajibabaee F, Peyrovi H, Haghani H, Bahrani N. The relationship between incidence and report of medication errors and working conditions. Int Nurs Rev. 2011;58(1):37-44.

20. Hajibabaee $F$, Joolaee S, Peyravi H, Alijany-Renany $H$, Bahrani N, Haghani $H$. Medication error reporting in Tehran: a survey. J Nurs Manag. 2014;22(3): 304-10.

21. Hartnell N, MacKinnon N, Sketris I, Fleming M. Identifying, understanding and overcoming barriers to medication error reporting in hospitals: a focus group study. BMJ Qual Saf. 2012;21(5):361-8.

22. Kagan I, Barnoy S. Factors associated with reporting of medication errors by Israeli nurses. J Nurs Care Qual. 2008;23(4):353-61.

23. Sanghera IS, Franklin BD, Dhillon S. The attitudes and beliefs of healthcare professionals on the causes and reporting of medication errors in a UK intensive care unit. Anaesthesia. 2007;62(1):53-61.

24. Kingston M, Evans S, Smith B, Berry J. Attitudes of doctors and nurses towards incident reporting: a qualitative analysis. http://www.mja.com.au/public/issues/ 181_01_050704/kin10795_fm.html [Internet]. 2004 [cited 2015 Jun 20]; Available from: https://digital.library.adelaide.edu.au/dspace/handle/2440/9952.

25. Almutary HH, Lewis PA. Nurses' willingness to report medication administration errors in Saudi Arabia. Qual Manag Health Care. 2012;21(3): 119-26.

26. Kim KS, Kwon S-H, Kim J-A, Cho S. Nurses' perceptions of medication errors and their contributing factors in South Korea: nurses' perceptions of medication errors. J Nurs Manag. 2011;19(3):346-53.

27. Güneş ÜY, Gürlek Ö, Sönmez M. Factors contributing to medication errors in Turkey: nurses' perspectives. J Nurs Manag. 2014;22(3):295-303.

28. Handler SM, Perera S, Olshansky EF, Studenski SA, Nace DA, Fridsma DB, et al. Identifying modifiable barriers to medication error reporting in the nursing home setting. J Am Med Dir Assoc. 2007;8(9):568-74.

29. Lin Y-H, Ma S. Willingness of nurses to report medication administration errors in southern Taiwan: a cross-sectional survey. Worldviews Evid-Based Nurs. 2009;6(4):237-45

30. Institute of Medicine (US) Committee on Quality of Health Care in America. In: Kohn LT, Corrigan JM, Donaldson MS, editors. To Err is Human: Building a Safer Health System [Internet]. Washington (DC): National Academies Press (US); 2000. [cited 2018 Mar 9]. Available from: http://www.ncbi.nlm.nih.gov/ books/NBK225182/.

31. Brady A-M, Malone A-M, Fleming S. A literature review of the individual and systems factors that contribute to medication errors in nursing practice. J Nurs Manag. 2009;17(6):679-97.

32. Petrova $E$, Baldacchino D, Camilleri M. Nurses' perceptions of medication errors in Malta. Nurs Stand. 2010:24(33):41-8.

33. Assefa F, Mosse A, Hailemichael Y. Assessment of clients' satisfaction with health service deliveries at Jimma University specialized hospital. Ethiop J Health Sci. 2011;21(2):101.

34. Bohomol E, Ramos LH, D'Innocenzo M. Medication errors in an intensive care unit. J Adv Nurs. 2009;65(6):1259-67.

35. Mayo AM, Duncan D. Nurse perceptions of medication errors: what we need to know for patient safety. J Nurs Care Qual. 2004;19(3):209-17.

\section{Submit your next manuscript to BioMed Central and we will help you at every step:}

- We accept pre-submission inquiries

- Our selector tool helps you to find the most relevant journal

- We provide round the clock customer support

- Convenient online submission

- Thorough peer review

- Inclusion in PubMed and all major indexing services

- Maximum visibility for your research

Submit your manuscript at www.biomedcentral.com/submit 